\title{
The use surface plasmon resonance to determine the optical parameters of UV-adhesive and control polymerization process
}

\begin{abstract}
Background: Ultraviolet adhesives are widely used in the manufacture of precision optical devices. It is known, that adding fillers to the glue reduces shrinkage after polymerization, reduces internal stresses, improves the reliability of the connection, and also allows you to control the optical properties of the connecting layer. By varying the amount and composition of the filler, you can change the refractive index of the compound, as well as improve the process ability of the compound due to faster polymerization.
\end{abstract}

Material and methods: To measure optical parameters of the studied samples, we used the following devices: spectrometer Plasmon-71, spectrophotometer Fluorotestnano2S, spectrophotometer UNICO 4802UV/VIS. As a source of UV radiation, we used semiconductor LED with the mean wavelength in its spectrum $406 \mathrm{~nm}$. As the objects for measurements, we used three samples, and each one represented the three-layered structure (glass-adhesive-glass) and, separately, three respective adhesives. All the glass elements of these samples were plates with the thickness $0.2 \mathrm{~mm}$ and dimensions $20 \times 20 \mathrm{~mm}$ from the glass with the refractive index $n=1.61$ with regard to vacuum. The sample differed between each other by composition of adhesives.

Results: The reflective index for each glue was defined. The speed of glue polymerization was corresponded to difference on a result changes the coefficient of reflection too. The highest velocity of polymerization was observed for the adhesive 3 (2100 arb.un./min), the lowest one - for the adhesive 1(300 arb.un./min), and adhesive 2(1200 arb.un./min). Thus, the polymerization velocity of the adhesive 3 is 7 times higher than that of the adhesive 1 and practically 2 times higher than for the adhesive 2. adhesives 2 and 3 are polymerized more uniformly over the whole sample depth. The reflection coefficient at the minimum SPR was measured. This characteristic corresponds to the absorption of the glue. It is shown that the adhesive composition No. 3 has the greatest absorption.

Conclusion: The obtained results show that the SPR method is informative and can be applied for investigations and optimization of UV-adhesive composition. Introduction of organosilicon acrylates with high dispersion as impurities in these adhesives enables to increase the velocity of adhesive polymerization.
Volume 3 Issue 2 - 2019

\author{
Hanna Dorozinska,' Glib Dorozinsky, \\ Volodymyr Maslov, ${ }^{2}$ Natalia Kachur ${ }^{2}$ \\ 'National Technical University of Ukraine "Igor Sikorsky Kyiv \\ Polytechnic Institute", Ukraine \\ ${ }^{2}$ V. Lashkaryov Institute of Semiconductor Physics NAS of \\ Ukraine, Ukraine
}

Correspondence: Volodymyr Maslov, V. Lashkaryov Institute of
Semiconductor Physics NAS of Ukraine, Nauki 4I, Kyiv, Ukraine, Tel +3804452558 30,Email maslov@isp.kiev.ua

Received: February 28, 2018 | Published: April 04, 2019

\section{Introduction}

Devices based on the phenomenon of surface plasmon resonance are characterized by high measurement accuracy, require a small sample volume of the test substance, and also have a high sensitivity to low concentrations of the test substances. Therefore, these devices are widely used for the study of liquids and gases in the chemical, pharmaceutical industry, medicine and ecology. Ultraviolet adhesives are widely used in the optical industry for joining optical components. Their widespread use is due to a number of advantages, namely: fast curing time, lack of heating process (allows reducing mechanical stresses), the ability to control the optical and strength properties of the compound by introducing fillers. Consequently, these adhesives have high manufacturability. The task of this work was: to establish the possibility of controlling the polymerization process using an instrument based on the surface plasmon resonance phenomenon, to establish the polymerization rate of experimental adhesive compositions and to determine the optical characteristics of adhesive joints.

\section{Materials and methods}

As the objects for measurements, we used three samples, and each one represented the three-layered structure (glass-adhesive-glass) and, separately, three respective adhesives. All the glass elements of these samples were plates with the thickness $0.2 \mathrm{~mm}$ and dimensions $20 \times 20 \mathrm{~mm}$ from the glass with the refractive index $n=1.61$ with regard to vacuum. The sample differed between each other by composition of adhesives. In all three cases, we used the adhesives that can be polymerized under action of UV radiation with the wavelengths from the range $340-380 \mathrm{~nm}$. In the samples, adhesive was preliminary polymerized. The sample 1 contained the UV adhesive without any impurities. The samples 2 and 3 contained the same adhesive but with addition of organosilicon acrylate, dispersion of which in the sample 3 was higher than in the sample 2. Previously ${ }^{1}$ spectrometer was used for polymerization process of UV-glue studying. This method is highinformative, but it is complicated and need much time for obtain result. To measure optical parameters of the studied samples, we used the following devices: 
I. Spectrometer Plasmon-71.

II. Spectrophotometer Fluorotestnano-2S.

III. Spectrophotometer UNICO 4802 UV/VIS.

As a source of UV radiation, we used semiconductor LED with the mean wavelength in its spectrum $406 \mathrm{~nm}$.

The values of refractive indexes for the studied UV-adhesives were measured using the spectrometer Plasmon-71, the optical setup of which is shown in Figure 1. 2,3

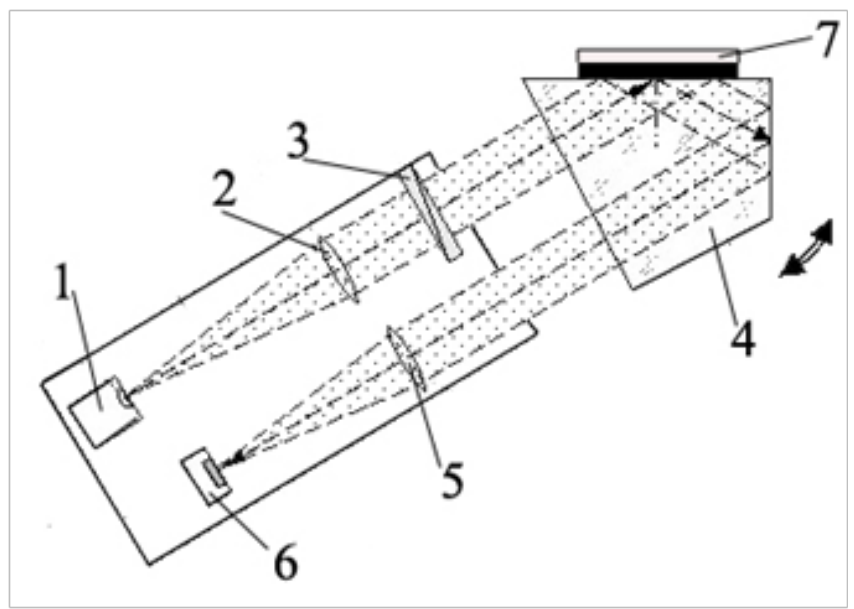

Figure I Optical setup of the spectrometer Plasmon-7I.

The laser diode $1(850 \mathrm{~nm})$ was located in the focus of the lenscollimator 2. Then, light passed through the polarizer 3 and to the glass optical prism 4 that had the refractive index $n=1.61$ (flint $\Phi 1$ ). The studied sample 7 was placed on the top surface of this prism. The optical contact of the sample with the prism 4 was provided by the immerse liquid $(n=1.61)$. Due to the lens 5 , the light reflected from the prism reached the sensitive surface of the detector 6 . The change in the angle of laser diode light incidence onto the sample surface was provided by turning the prism. The sample represented a glass plate of $1 \mathrm{~mm}$ thick and with dimensions $20 \times 20 \mathrm{~mm}$ from the glass $\Phi 1(\mathrm{n}=1.61)$, the surface of which was preliminary covered with the plasmon-carrying gold layer with the thickness close to $50 \mathrm{~nm}$. In its turn, this layer was covered with the UV-adhesive (1, 2 and 3 , respectively). At the initial stage, we measured the dependence of reflection coefficient (for the laser light beam reflected from the sample surface) on the angle of light incidence on the sample with non-polymerized adhesive. It enabled us to determine characteristics of surface plasmon resonance (SPR), namely: the position of minimum for the curve of SPR and its half width as well as the angle of total internal reflection (TIR) at the boundary "adhesive - air". Then, using digital processing of results we determined the refractive index of the non-polymerized adhesive. In what followed, we performed the process of adhesive polymerization by irradiating it with UV light of the semiconductor LED for $20 \mathrm{~min}$. During this process, we carried out measurements of the kinetics inherent to the shift of SPR curve by tracing the dependence of reflection coefficient on time in the definite point on the left slope of SPR curve, i.e., for the fixed angle of laser light incidence onto the sample. This point was chosen in the linear part of the SPR curve. After completion of the polymerization process, we again measured the dependence of reflection coefficient on the angle of light radiation incidence onto the sample with polymerized adhesive and performed the procedure of determining the refractive index of the polymerized adhesive. All the results of measurements were processed using the specially developed software and kept in the memory of PC. Thermo boxing was used to stabilize the temperature. ${ }^{4-7}$ This helped reduce the measurement error.

Investigation of characteristics inherent to the transmitted light passing through the studied samples (three-layered structures "glass - adhesive - glass") was performed using the spectrophotometer UNICO 4802UV/VIS having the operation range of wavelength scanning $190 \ldots 1100 \mathrm{~nm}$. Before each measurement, we carried out calibration. The step of measurements was $0.5 \mathrm{~nm}$. The measurements were performed in the regime for determining absorption (regime A). This spectrophotometer has two channels: the reference (basic) and measuring ones. The result of measurement is the difference of signals at the output of both channels. It means that there performed is comparison of light passage through these channels. At first, we measured absorption of the glass substrate relatively to air. In this case, this substrate was placed into the measuring channel, while the reference channel contained only air. Then, the measured glass substrate was placed into the reference channel, and the measuring channel was sequentially filled with the three samples of adhesives. Thus, we measured the adhesive absorption relatively to the glass. Investigation of fluorescence characteristics inherent to the studied samples (three-layered structures "glass - adhesive - glass") was performed using the spectrophotometer Fluorotestnano-2S with the operation wavelength range $313 \ldots 900 \mathrm{~nm}$. The scanning step was $0.322 \mathrm{~nm}$. As the exciting radiation sources, we used LEDs with the wavelengths 463,515 and $631 \mathrm{~nm}$. At the very beginning, we measured fluorescence of the glass substrate without any adhesive. Then, this operation was repeated with the samples of adhesives. Before each measurement, we carried out calibration. In the spectrophotometer Fluorotestnano 2S, the optical axes of the source and detector of radiation are allocated at the angle $90^{\circ}$ relatively to each other, while the studied sample - at the angle $45^{\circ}$ both relatively to the radiation source and to detector. It gives the possibility to fix the light reflected from the sample. The results obtained using the spectrophotometers were processed by the specially developed program and kept in PC.

\section{Results and discussion}

The measured dependences of the reflection coefficient $\mathrm{R}$ on the angle of incidence of laser radiation onto the samples with nonpolymerized and polymerized adhesives are shown in Figure 2. Represented in Figure 2A are the reflection curves for three adhesives. The V-like part of reflection curves is the SPR curve. This part can be characterized by the angular position and reflection coefficient in the curve minimum. The angular position enables to determine the refraction index of the substance studied (adhesive, in our case), and the reflection coefficient in the curve minimum - the adhesive absorption. As seen from Figure 2A, within the range of angles 64.5 ...66 degrees there are Brewster's angles (TIR). The level of $R$ within the range of angles $66 \ldots 68$ degrees also indicates the adhesive absorption. Therefore, it should be concluded that the adhesive 3 has higher absorption, although it possesses lower refraction index at the same time. The kinetics of polymerization process is shown in Figure 3. It describes time changes in reflection coefficient values for three samples. The initial value of this quantity corresponded to the point on the left slope of the SPR curve for each adhesive before polymerization. This point is related with the definite angle of incidence of the laser beam onto the boundary "gold - adhesive". During polymerization, the SPR curve shifted to the right. As a result, 
the chosen point rose up along the slope of the curve, and the value of reflection coefficient increased. Each adhesive polymerizes with a different velocity. It is seen via the slope of kinetic curves. The highest velocity of polymerization was observed for the adhesive 3(2100 arb.un./min), the lowest one - for the adhesive 1(300 arb. un./min), and adhesive 2(1200arb.un./min). Thus, the polymerization velocity of the adhesive 3 is 7 times higher than that of the adhesive 1 and practically 2 times higher than for the adhesive 2 . Numerical calculations enabled to determine indexes of refraction $\mathrm{n}$ for adhesives before and after polymerization (Table 1). Adduced in this table are the values of Brewster angles $(\Theta Б)$ as well as angular positions of SPR curves minima ( $\Theta \mathrm{min}$ ) expressed in angular degrees before and after polymerization. Also, there adduced are relative augmentations in percents of the angle values $(\delta \Theta \min , \delta \Theta Б)$ as well as absolute augmentations of the adhesive refraction index $\Delta \mathrm{n}$.

Table I Results of measurements of refraction indexes

\begin{tabular}{|c|c|c|c|c|c|c|c|c|c|}
\hline \multirow[b]{2}{*}{$\begin{array}{l}\text { No of the } \\
\text { sample }\end{array}$} & \multicolumn{3}{|c|}{ Before polymerization } & \multicolumn{3}{|c|}{ After polymerization } & \multicolumn{2}{|c|}{ Relative change, $\%$} & \multirow[b]{2}{*}{$\begin{array}{l}\Delta n \\
\times 10^{-3}\end{array}$} \\
\hline & $\begin{array}{l}\text { Omin } \\
\text { deg }\end{array}$ & $\begin{array}{l}\Theta Б \\
\text { deg }\end{array}$ & $\mathbf{n}$ & $\begin{array}{l}\Theta \min \\
\operatorname{deg}\end{array}$ & $\begin{array}{l}\Theta Б \\
\text { deg }\end{array}$ & $\mathbf{n}$ & $\delta \Theta \min$ & $\delta \Theta Б$ & \\
\hline 1 & 70.82 & 65.78 & 1.4615 & 70.84 & 65.82 & 1.4628 & 0.03 & 0.06 & 1.3 \\
\hline 2 & 70.77 & 65.7 & 1.4602 & 73.42 & 68.22 & 1.4816 & 3.75 & 3.83 & 21.4 \\
\hline 3 & 70.11 & 64.99 & 1.4507 & 71.13 & 65.95 & 1.4655 & 1.46 & 1.48 & 14.8 \\
\hline
\end{tabular}

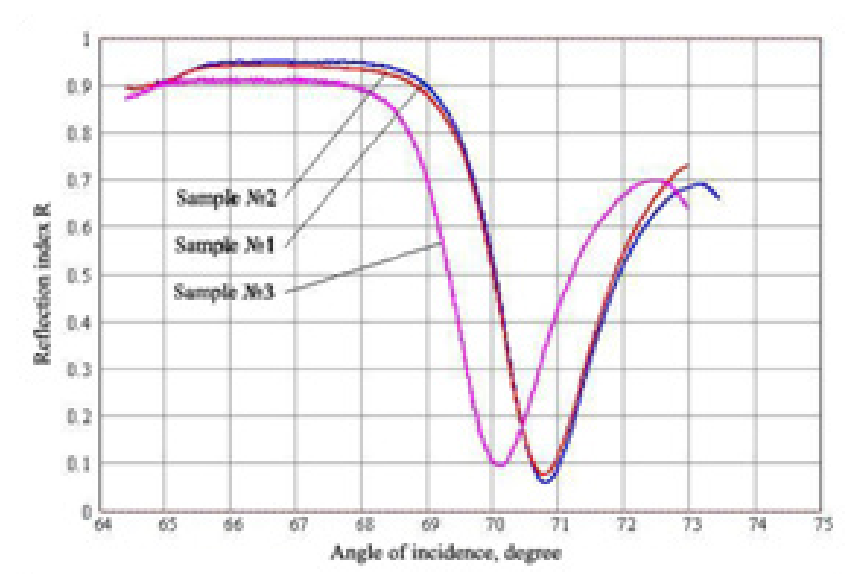

A

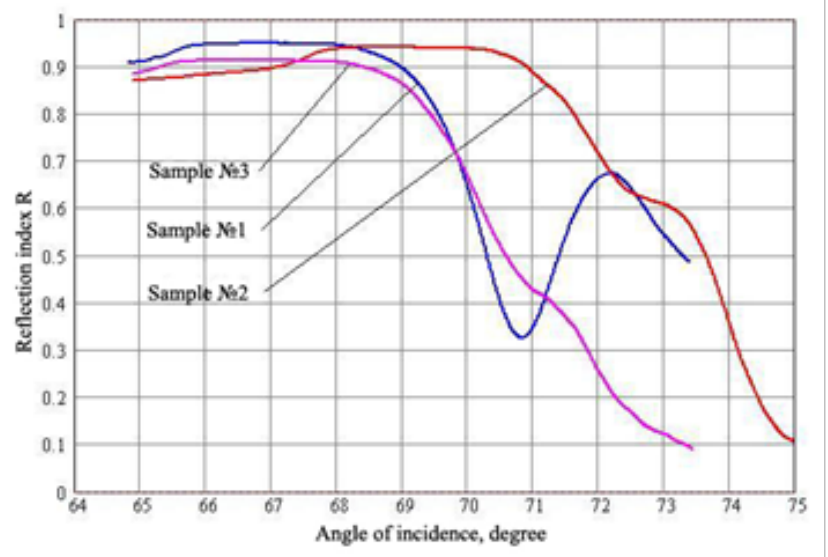

B

Figure 2 SPR reflection curves for non-polymerized $(A)$ and polymerized $(B)$ states of UV-solidified adhesives.

The results shown in Table 1 enabled to draw the conclusion that adhesives 2 and 3 are polymerized more uniformly over the whole sample depth. Therefore, the shifts of the SPR curve minimum and Brewster angle are practically the same, contrary to the case of the adhesive 1. It is related with that the SPR field can "feel" changes in the refraction index only at the distance from the sensitive surface (gold layer) lower than the halved wavelength of exciting radiation, which in our case is close to $425 \mathrm{~nm}$. In its turn, the Brewster angle (TIR angle) is defined by the refraction index at the boundary of media gold - adhesive, which thickness is several orders lower than $425 \mathrm{~nm}$. Thereof, one can conclude that the polymerization velocity depends on the surface state or availability of polymerization nuclei. In the adhesives 2 and 3 , the role of these polymerization nuclei is played by organosilicon acrylates, while for the adhesive 1 this role is performed by the boundary gold - adhesive. The total area of polymerization nuclei surface influences on the polymerization velocity, which is confirmed by the plots in Figure 3. The higher polymerization velocity in the sample 3 can be explained by the fact that the acrylate impurity in it was of higher dispersion. The results of measurements of the absorption coefficients inherent to the structures glass - adhesive - glass within the range $200 \ldots 400 \mathrm{~nm}$ are shown in Figure 4A and within the range 400...900nm in Figure 4B. The spectra of fluorescence inherent to the structures glass - adhesive glass within the range $400 \ldots 700 \mathrm{~nm}$ are shown in Figure 5 Figure
$4 \&$ Figure 5 show that the adhesive 1 has the highest transparency (i.e., the lowest absorbance) within the visible range of wavelengths $(400 \ldots 750 \mathrm{~nm})$ and relatively low level of the fluorescence intensity. It is related with not only absence of organosilicon acrylate impurities but also with a higher content of the monomer-diluent, as compared with the other adhesives. The adhesive 2 has the highest absorption in the visible range and the lowest absorption in the UV-range $(190 \ldots 400 \mathrm{~nm})$.

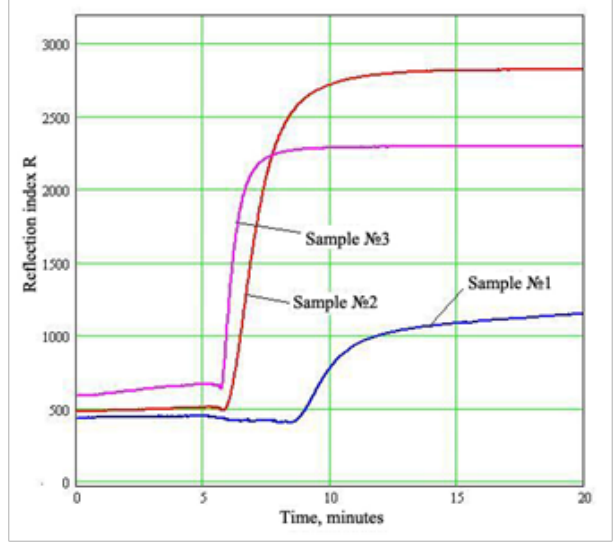

Figure 3 Polymerization kinetic curves inherent to UV-adhesives. 


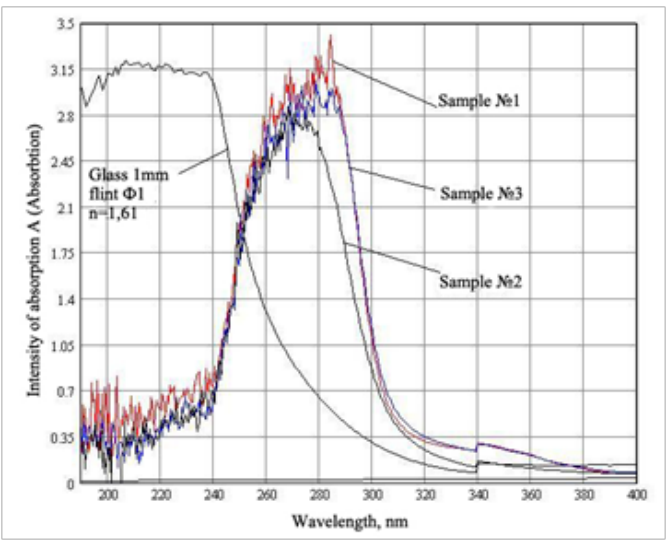

A

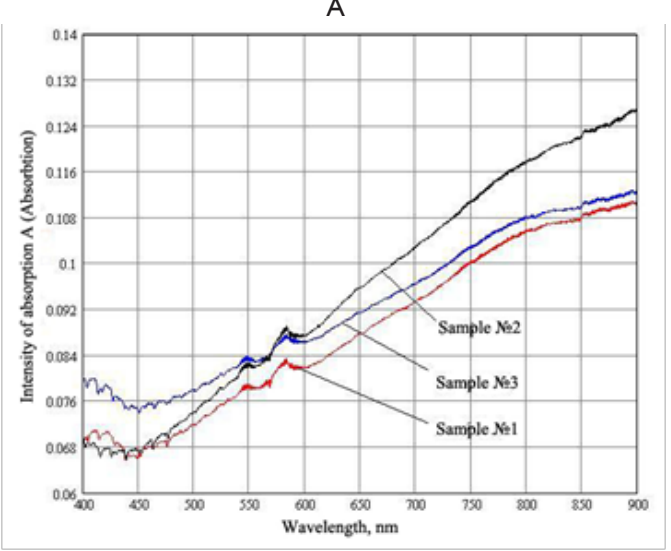

B

Figure 4 Absorption characteristics for the adhesives and glass.

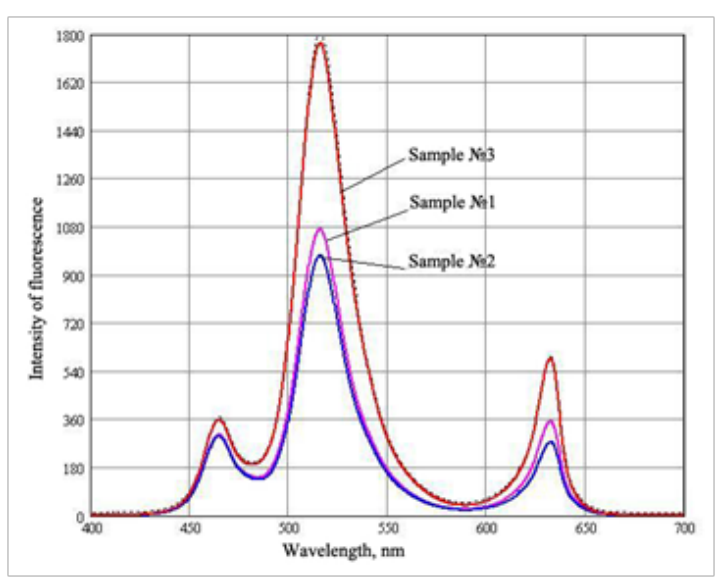

Figure 5 Fluorescence of the structures glass - adhesive - glass.

\section{Conclusion}

The obtained results show that the SPR method is informative and can be applied for investigations and optimization of UV-adhesive composition. Adding of organosilicon acrylates with high dispersion as impurities in these adhesives enables to increase the velocity of adhesive polymerization.

\section{Acknowledgments}

None.

\section{Conflicts of interest}

Author declares that there is no conflicts of interest.

\section{References}

1. Decker Ch. Kinetic Study and New Applications of UV Radiation Curing. Macrmolecular rapid communications. 2003;23(18):1067.

2. http://plasmon.org.ua/www.plasmon.org.ua

3. Gridina N, Dorozinsky G, Khristosenko R, et al. Surface Plasmon Resonance Biosensor. Sensors and Transducers. 2013;149(2):60-68.

4. Dorozinsky G, Maslov V, Samoylov A, et al. Reducing measurement uncertainty of instruments based on the phenomenon of surface plasmon resonance. American Journal of Optics and Photonics. 2013;1(3):17-22.

5. Yurish SY. Sensors and Applications in Measuring and Automation Control Systems. Advances in Sensors: Reviews. International Frequency Sensor Association Publishing; 2016.

6. Homola J. Surface Plasmon Resonance Based Sensors. Springer-Verlag; 2006.

7. Özdemir SK, Turhan-Sayan G. Temperature effects on surface plasmon resonance: Design considerations for an optical temperature sensor. Journal of Lightwave Technology. 2003;21(3):805-814. 